\title{
A hierarchical location model for public facility planning
}

\author{
João C. Teixeira *, António P. Antunes \\ Civil Engineering Department, University of Coimbra, 3030-290 Coimbra, Portugal
}

Received 4 May 2006; accepted 18 December 2006

Available online 14 January 2007

\begin{abstract}
In this article, we present a discrete hierarchical location model for public facility planning. The main features of the model are: an accessibility maximization objective; several levels of demand and of facilities; a nested hierarchy of facilities (i.e. a facility of a given level can serve demand of equal and lower levels); maximum and minimum capacity constraints; and user-to-facility assignment constraints. The latter include single-assignment and closest-assignment constraints, as well as a new type of constraints called path-assignment constraints. Their purpose is to enforce some desirable properties for the spatial pattern of assignments. If they are not included, model solutions are difficult to interpret and to explain in a public facility planning context, therefore being less likely to be accepted by the users. The usefulness of the model is illustrated through a real-world application to school network planning.
\end{abstract}

(C) 2007 Elsevier B.V. All rights reserved.

Keywords: Location; Integer optimization; Assignment constraints; Public facility planning; School network

\section{Introduction}

During the last two decades, planning processes involving public facilities such as schools or hospitals became increasingly more complex, especially because of the participatory mechanisms they started to involve. Indeed, in the presence of stakeholders with different viewpoints and interests, planning solutions can only be widely agreed upon if they are the result of transparent, rational planning processes. When the number of possible planning solutions is very large, optimization models are

\footnotetext{
* Corresponding author. Tel.: +351 239 797139; fax: +351 239 797146.

E-mail addresses: jtex@dec.uc.pt (J.C. Teixeira), antunes@ dec.uc.pt (A.P. Antunes).
}

indispensable decision-aid tools. Location models certainly are among the main optimization models to be used within public facility planning processes. These models are basically aimed at determining the most efficient locations for all types of facilities according to some objective or objectives (cost minimization, accessibility maximization, etc.). They are classified as continuous or discrete depending on whether the facilities can be located anywhere on the plane or in some points of the plane, specified in advance. In practical applications, planners often resort to discrete location models.

Location models have been extensively studied since the 1960s, in the operational research, management science, industrial engineering, economic geography and spatial planning literatures. ReVelle and Eiselt (2005) present a concise review of the 
main classes of continuous and discrete location models. ReVelle (1987) and Marianov and Serra (2002) discuss discrete models for public facility location. Daskin (1995) presents a didactic textbook on modeling and solving discrete location models. Labbé and Louveaux (1997) review specialized solution methods for basic and extended discrete location models.

In this article, we present a discrete hierarchical location model for public facility planning, considering several levels of demand and several types of facilities. The model is an extension of the wellknown $p$-median model, which applies to facility location problems where the objective is to maximize the accessibility of users to facilities.

The model was developed within the framework of the Coimbra Educational Charter 2006-2015, to help making decisions on the redeployment of the primary school network of the municipality of Coimbra, Portugal. By law, all Portuguese municipalities must have an educational charter where the infrastructure, equipment, human, and financial resources necessary for preschool, primary and secondary education are specified. The preparation of the educational charter of a municipality is advised by the education council of the municipality. This body integrates, among others, representatives of the local administration, the Ministry of Education, private school owners, public school administrations, teacher unions, and student parents, which often have different viewpoints and interests with regard to the evolution of school networks.

This article is organized as follows. In Section 2, we present the basic location models applicable when the objective is to maximize the accessibility of users to facilities. These models consider a single level of demand and a single type of facility. In Section 3, we discuss different user-to-facility assignment constraints, including a new type of constraints called path-assignment constraints. In Section 4 , we present the hierarchical location model. In Section 5, we discuss the results obtained with this model for Coimbra's primary school network. Finally, in Section 6, we summarize the main contributions of the article, reflect on the application of the model in Coimbra, and identify some research needs to be fulfilled in the future.

\section{Basic models}

In this section, we present the basic location models upon which the hierarchical model is built.
The simplest of these models is the $p$-median model (ReVelle and Swain, 1970), which can be stated as follows. We are given a set of demand centers $I=\{1, \ldots, n\}$, where each center $i$ has a demand $u_{i}$ (number of users), a set of sites $J=\{1, \ldots, m\}$, and travel costs $c_{i j}$ for serving all the demand from center $i$ at site $j$. Travel costs are defined as $c_{i j}=u_{i} \cdot d_{i j}$, where $d_{i j}$ is the unit travel cost between center $i$ and site $j$ (or distance, if the unit cost is constant). The problem is to find the set of $p$ facilities that should be open, and to determine which centers should be served from which facilities, so that the travel costs of serving all the demand from all centers is minimized. For formulating the model, we define two sets of decision variables: binary location variables $y_{j}$, where $y_{j}=1$ if a facility is located (or "open") at site $j \in J$, and $y_{j}=0$ otherwise; and assignment variables $x_{i j}$ representing the fraction of the demand from center $i \in I$ served at site $j \in J$. The formulation of the $p$-median model is as follows:

(PM):

$$
\begin{aligned}
\text { Minimize } & \sum_{i \in I} \sum_{j \in J} c_{i j} x_{i j} \\
\text { Subject to : } & \sum_{j \in J} x_{i j}=1, \quad \forall i \in I, \\
& x_{i j} \leqslant y_{j}, \quad \forall i \in I, j \in J, \\
& \sum_{j \in J} y_{j}=p, \\
& 0 \leqslant x_{i j} \leqslant 1, \quad \forall i \in I, j \in J, \\
& y_{j} \in\{0,1\}, \quad \forall j \in J .
\end{aligned}
$$

The objective function (1) of this mixed-integer optimization model expresses the minimization of travel costs, which can be seen as a proxy for accessibility maximization. Constraints (2) state that all centers have to be fully served. Constraints (3) link location and assignment decisions by stating that centers can only be assigned to an open facility. Constraint (4) sets the number of open facilities equal to parameter p. Finally, constraints (5) and (6) define decision variables.

Optimal solutions of (PM) have the so-called single assignment and closest assignment properties (Krarup and Pruzan, 1983), that is, centers are fully served by the closest open facility (or, if travel cost is not monotonically dependent on distance, the least travel cost facility). This happens because, as there are no capacity constraints, nothing is gained by splitting the demand from a given center across 
several facilities, and the objective forces centers to be assigned to the closest (least cost) facility.

The second basic model is derived from (PM) by adding constraints on the minimum and maximum capacity of facilities and deleting the constraint on the number of open facilities. Note that, with the capacity constraints, the number of open facilities becomes an output of the model rather than a parameter. Let $b_{j}$ and $B_{j}$ be the minimum and maximum capacity for a facility to be open at site $j$. This model, denoted capacitated median model, is formulated as follows:

$(\mathrm{CM})$ :

$$
\begin{aligned}
\text { Minimize } & \sum_{i \in I} \sum_{j \in J} c_{i j} x_{i j} \\
\text { Subject to: } & \sum_{j \in J} x_{i j}=1, \quad \forall i \in I, \\
& x_{i j} \leqslant y_{j}, \quad \forall i \in I, \quad j \in J, \\
& \sum_{i \in I} u_{i} x_{i j} \geqslant b_{j} y_{j}, \quad \forall j \in J, \\
& \sum_{i \in I} u_{i} x_{i j} \leqslant B_{j} y_{j}, \quad \forall j \in J, \\
& \sum_{k \in J \mid d_{i k} \leqslant d_{i j}} x_{i k} \geqslant y_{j}, \quad \forall i \in I, j \in J, \\
& x_{i j} \in\{0,1\}, \quad \forall i \in I, j \in J, \\
& y_{j} \in\{0,1\}, \quad \forall j \in J .
\end{aligned}
$$

Expressions (10) and (11) are, respectively, the minimum and maximum capacity constraints. As the closest assignment and single assignment properties of solutions do not hold for capacitated models, they are enforced explicitly with constraints (12) and (13), respectively. Closest assignment constraints (12) work as follows. For any center $i$ and site $j$, if $y_{j}=0$ then the constraint has no effect; if $y_{j}=1$ then center $i$ must be fully served from the facility located at site $j$ or from a facility at the same or lower distance. Gerrard and Church (1996) thoroughly review formulations and applications of closest assignment constraints in several location models arising in the public and private sectors. Although the authors note that a different formulation, the so-called Rojeski-ReVelle (RR) constraints, is frequently used in the literature, we opted for constraints (12) for two reasons. First, unlike RR constraints, constraints (12) remain valid if a given center has two or more equidistant facilities that are the closest. Second, constraints (12) provide a tighter linear relaxation, as RR constraints are implied by (12) together with (9); thus, the model can be solved more efficiently with an integer optimization algorithm based on linear relaxations. Finally, constraints (13) force assignment variables to be binary. We remark that if all centers have a single closest facility then constraints (12) will force assignment variables to be integer, even if (13) is relaxed. Otherwise, if a center has two or more equidistant facilities that are the closest, constraints (12) allow demand to be freely distributed among those facilities and (13) is necessary to impose single assignment.

Unlike the $p$-median model, the capacitated median model has rarely been dealt with in the literature. Carreras and Serra (1999) use it without the maximum capacity constraints to represent a pharmacy location problem in a rural region, and solve it through a tabu search heuristic. Verter and Lapierre (2002) employ a similar model for locating preventive health care facilities with the objective of maximizing population coverage, and solve it with a commercial optimizer. Kalcsics et al. (2002) use model (CM) with constraint (4) for designing balanced and compact sales territories, and solve it through a variable neighborhood search heuristic.

\section{Assignment constraints}

In this section, we analyze the spatial pattern of user-to-facility assignments resulting from different assignment constraints. As noted previously, solutions to location models including capacity constraints do not have the single and closest assignment properties. This may happen because facilities have limited capacity, and thus users are diverted to other facilities, or users are "captured" to ensure the minimum capacity of a facility. In a public facility planning context, it should be prevented that users from the same center are split among different facilities; that users from neighboring centers are assigned to different facilities; that users are assigned to a distant facility when there are closer open facilities; that the path traveled by users to the facility they are assigned to crosses a center assigned to a different facility. If these conditions are violated, solutions are difficult to interpret by decision-makers and to explain to users, and will certainly be difficult to implement in practice. Gerrard and Church (1996) make a similar argument in their article, where they recommend the use of closest assignment constraints in capacitated location 
models for public facility planning because they are likely to improve public confidence and acceptance in the corresponding solutions.

Our analysis focuses on three types of constraints: the closest assignment constraints (12); the single assignment constraints (13); and a new type of constraints designated as path assignment constraints (15). The latter are an alternative to closest assignment constraints. Although they do not require centers to be assigned to the closest facility, they guarantee that, if a center is assigned to a given facility, all centers "near" the "path" traveled by the users to reach the facility must also be assigned to it. The definition of "near" depends on the context of application of the model. The formulation of path assignment constraints is as follows:

$\sum_{k \in P_{i j}} x_{k j} \geqslant\left|P_{i j}\right| \cdot x_{i j}, \quad \forall i \in I, j \in J$

where $P_{i j}$ is the subset of centers $k \in I$ that are "near" the shortest path from $i$ to $j$, and $\left|P_{i j}\right|$ is the cardinality of this set. Expression (15) states that if $x_{i j}=1$ then $x_{k j}=1$ for all $k \in P_{i j}$.

Some constraints used in districting models are similar to the path assignment constraints. These models apply to the partitioning of a set of spatial units (i.e. city blocks, census tracts, or other geographic entities) into subsets, called districts, according to some objective. Like with location models, spatial units are represented with discrete centers connected through an underlying network. Desired properties of districts often include compactness and contiguity, i.e. they should be round shaped rather than spread out, and should be connected. Kalcsics et al. (2002) use model (CM) for sales districting, including closest assignment in order to produce connected districts. Zoltners and Sinha (1983) formulate a model for sales districting, where district centers (i.e. the seed units for the districts) are predefined. Considering binary decision variables $x_{i j}=1$ if unit $i$ is assigned to district $j$, and zero otherwise, contiguity is enforced with constraints

$x_{i j} \leqslant \sum_{k \in A_{i j}} x_{k j}, \quad \forall i \in I, j \in J$,

where $A_{i j}$ is the set of units $k \in I$ that immediately precede unit $i$ on a shortest path from district center $j$ to unit $i$. Expression (16) states that unit $i$ can be assigned to district $j$ only if at least another unit, adjacent to $i$ on a path connecting to the district center, is also assigned to $j$. In order to reduce the "rigidity" of assignments (as building districts along shortest paths guarantees contiguity but rules out some contiguous configurations), the authors propose augmenting sets $A_{i j}$ by either considering the second, third, etc., shortest paths, or by manual modification by an expert user. A strict shortestpath approach is adopted by Mehrotra et al. (1998) to ensure contiguity in a political districting model. Caro et al. (2004) present a model for school districting (with predefined school locations) including constraints similar to (16), where sets $A_{i j}$ are defined as the set of units $k \in I$ that are adjacent to unit $i$ and are closer to district center $j$ than to unit $i$ (but not necessarily on the shortest path).

The impact of including the different assignment constraints in a location model will be illustrated with one of the random instances used to test model $(\mathrm{CM})$. The instances were built as follows. First, a set of $n=20$ centers was generated uniformly in the unit square and their coordinates scaled by 1000. Sites were assumed to be coincident with centers. Then a planar network was created by computing the Delaunay triangulation (Weisstein, 1999), with edge length set equal to the Euclidean distance. Distances $d_{i j}$ were computed by finding all shortest paths on the resulting network. Demands $u_{i}$ were generated uniformly in the interval 5-95. Minimum capacities $b_{j}$ were set to 200 for all sites. Maximum capacities $B_{j}$ were set to the total demand (i.e. they are not binding). Finally, path assignment sets $P_{i j}$ were created by adding all centers in the shortest path from $i$ to $j$, plus all centers within a radius of 100 from any node in this path. Panel (i) of Fig. 1 shows the network and demands of the test instance. As the total demand is 809 , at most four facilities can satisfy the minimum capacity of 200 .

This instance was solved with four variants of model (CM): without assignment constraints, i.e. (12) removed and (13) relaxed; with single assignment only, i.e. (12) removed; with single + path assignment, i.e. (12) replaced by (15); and with single + closest assignment, i.e. the full model (CM). Solutions are shown in Fig. 1 (where numbers in panels (ii)-(vi) refer to capacity). The $p$-median model (PM) solution is included for comparison, with $p=4$. In this solution, centers are fully assigned to the closest facility, but one of the facilities does not satisfy the minimum capacity constraints. On the other hand, all solutions of variants of model (CM) satisfy minimum capacities, but some undesirable user-to-facility assignments occur if path or closest assignment constraints are not imposed. Without any assignment constraints, the demand 


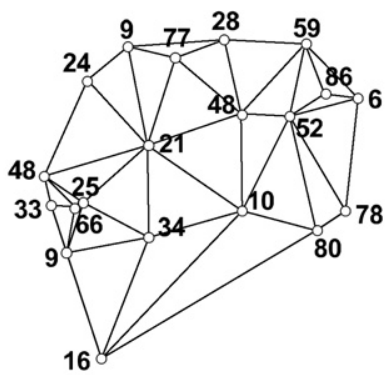

(i) network and demands

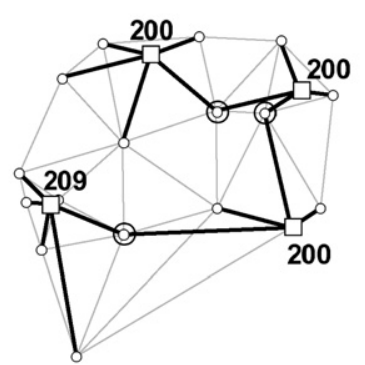

(iii) Model (CM), no assignment constraints

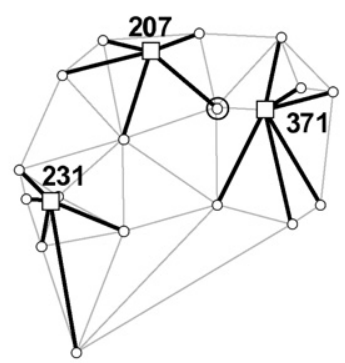

(v) Model (CM), single+path assignment

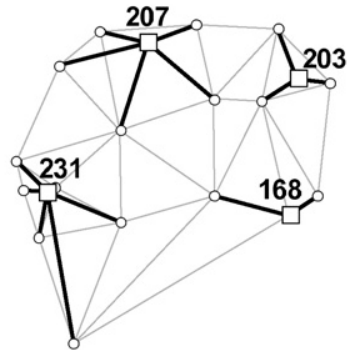

(ii) p-median model

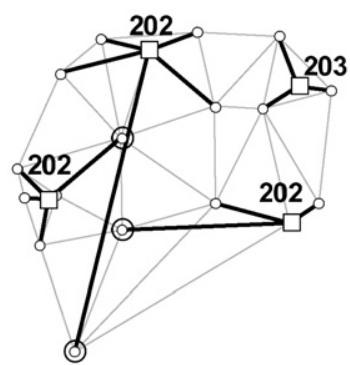

(iv) Model (CM), single assignment only

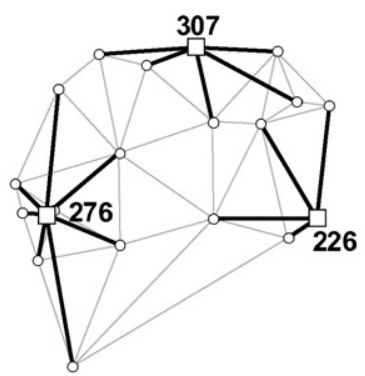

(vi) Model (CM), single+closest assignment

Fig. 1. Solutions for random instance.

from some centers is split among two facilities. With single assignment only, split demands are eliminated but some centers are assigned to a facility much further than the closest. Adding path or closest assignment constraints eliminates these undesirable patterns (although with path assignment one of the centers is not assigned to the closest facility). As expected, adding more constraints degrades the optimal objective value (Table 1). In addition, in this example, solutions with path or closest assignment constraints have fewer open facilities. This can be expected to occur frequently because the number of feasible solutions diminishes. That is, path and closest assignment constraints prevent dis-
Table 1

Solutions for random instance

\begin{tabular}{lll}
\hline Model & $\begin{array}{l}\text { Number of open } \\
\text { facilities }\end{array}$ & $\begin{array}{l}\text { Relative objective } \\
\text { value }(\%)\end{array}$ \\
\hline $\begin{array}{l}p \text {-median } \\
(\mathrm{CM}), \text { no assignment }\end{array}$ & 4 & 83 \\
$\quad$ constraints & & 91 \\
$\begin{array}{l}\text { (CM), single } \\
\quad \text { assignment only }\end{array}$ & 4 & 100 \\
$\begin{array}{l}\text { (CM), single + path } \\
\quad \text { assignment }\end{array}$ & 3 & 138 \\
$\begin{array}{l}\text { (CM), single + closest } \\
\text { assignment }\end{array}$ & 3 & 146 \\
\end{tabular}


advantageous assignments of some small centers for the sake of the global optimum. The resulting solutions are easier to interpret and to explain in a planning context, therefore being more likely to be accepted by the users.

\section{Hierarchical model}

In this section we present a hierarchical version of the capacitated median problem of Section 2, considering several levels of demand and several types (or levels) of facilities. Specifically, the demand of centers is discriminated in levels, which must be separately assigned to facilities capable of providing them. We consider a nested (or successively inclusive) hierarchy of facilities where a level-s facility $(s=1,2, \ldots, n s)$ can serve demands of level $1, \ldots, s$. Examples of nested hierarchical facilities arise in the context of public facility planning. A typical example is a health care network composed of local health care units, providing basic services, and central hospitals providing both basic and specialized services. Another example is an education network, composed of kindergartens, primary schools and secondary schools, in which a higher level of education can only be located at a site if all lower-levels are also located there. As noted by Weaver and Church (1991), the various levels of facilities may or may not be physically distinct (in the example of schools, these could be separate buildings located in the same community). Other examples of hierarchical facilities arising in postal and banking services are described by Daskin (1995).

Reviews and classifications of hierarchical models are provided by Narula (1986) and Church and Eaton (1987), focusing on models with distance minimization and with coverage maximization objectives, respectively. Before introducing our model, we will briefly describe some representative examples of hierarchical extensions of the $p$-median model. Weaver and Church (1991) formulate a model with a nested facility hierarchy with any number of levels. The model does not include capacity constraints, thus an optimal solution can always be found with the single and closest assignment properties. The model is solved with Lagrangian relaxation combined with an interchange heuristic, and results are reported for two test networks, with two and three levels. Galvão et al. (2006) formulate a model with a nested facility hierarchy with three-levels, addressing a real-world health care application. The model allows service referrals (that is, a fraction of demand served at a lower-level facility may be referred directly to a higher-level facility) and includes capacity constraints (however, single-assignment is not enforced and split assignments can occur). The authors propose a Lagrangian heuristic to solve the model. Eitan et al. (1991) formulate a model with nested or more general facility hierarchies (with any number of levels), capacity constraints, and service referrals. The model is applied to several problems appearing in the literature and is solved with a commercial integer optimizer.

Relatively to hierarchical models in the literature, the model we present here is the first to combine capacity constraints (minimum and maximum) with assignment constraints such as closest- or pathassignment (in addition to single-assignment). Consider the following additional or revised notation for data: $S=\{1, \ldots, n s\}$ is the set of demand levels (and of facility types); $u_{i s}$ is level-s demand of center $i ; B_{j s}$ and $b_{j s}$ are the maximum and minimum capacities of a type-s facility at site $j ; J_{s}^{0}$ is the set of sites with existing type- $s$ facilities; $p_{s}$ is the maximum numbers of new type- $s$ facilities to open; $q_{s}$ is the maximum numbers of existing type-s facilities to close; $D_{s}$ is the maximum user-to-facility distance for demand level $s$. Decision variables are defined as follows: $x_{i j s}$ is the fraction of the level- $s$ demand of center $i$ satisfied by a facility located at site $j ; y_{j s}=1$ if a type- $s$ facility is located at $j$, and equals zero otherwise; $z_{j s t}$ is the capacity occupied with demand level $s$ of a level- $t$ facility located at $j$. The hierarchical capacitated median model is formulated as follows:

(HCM):

$$
\begin{aligned}
\text { Minimize } & \sum_{i \in I} \sum_{j \in J} \sum_{s \in S} d_{i j} u_{i s} x_{i j s} \\
\text { Subject to: } & \sum_{j \in J} x_{i j s}=1, \quad \forall i \in I, s \in S, \\
& x_{i j s} \leqslant \sum_{t \in S \mid t \geqslant s} y_{j t}, \quad \forall i \in I, j \in J, s \in S, \\
& \sum_{t \in S \mid t \geqslant s} z_{j s t}=\sum_{i \in I} u_{i s} x_{i j s}, \quad \forall j \in J, s \in S, \\
& \sum_{s \in S \mid s \leqslant t} z_{j s t} \geqslant b_{j t} y_{j t}, \quad \forall j \in J, t \in S, \\
& \sum_{s \in S \mid s \leqslant t} z_{j s t} \leqslant B_{j t} y_{j t}, \quad \forall j \in J, t \in S, \\
& \sum_{k \in J \mid d_{i k} \leqslant d_{i j}} x_{i k s} \geqslant y_{j t}, \quad \forall i \in I, j \in J, \\
& s \in S, \forall t \in S \mid t \geqslant s,
\end{aligned}
$$




$$
\begin{aligned}
& \sum_{j \in J \backslash J_{s}^{0}} y_{j s} \leqslant p_{s}, \quad \forall s \in S, \\
& \sum_{j \in J_{s}^{0}} y_{j s} \geqslant\left|J_{s}^{0}\right|-q_{s}, \quad \forall s \in S, \\
& x_{i j s}=0, \quad \forall i \in I, j \in J, s \in S \mid d_{i j}>D_{s}, \\
& x_{i j s} \in\{0,1\}, \quad y_{j s} \in\{0,1\}, \quad z_{j s t} \geqslant 0, \\
& \forall i \in I, j \in J, s \in S, t \in S .
\end{aligned}
$$

Constraints (18) ensure that all demands of all levels from all centers are satisfied. Constraints (19) impose that a given level of demand can only be satisfied by a facility of equal or higher level. Constraints (20) define capacity variables $z_{j s t}$ by stating that the demand of each level assigned to a site has to be served by some facility of equal or higher level located there. Constraints (21) and (22) impose maximum and minimum limits on capacity, according to facility type. With this formulation, capacity is shared by all demand levels. Additional constraints could easily be added to impose separate capacity limits per demand level, as the model already includes capacity variables $z_{j s t}$ discriminating the demand levels. Note that constraints (19) are redundant for the integer formu- lation (that is, the same set of integer solutions is obtained if they are removed), as constraints (20) and (22) allow a variable $x_{i j s}$ for a given $s \in S$ to be non-zero only if there is a variable $y_{j t}$ equal to one for some $t \geqslant s$. However, (19) are kept in the formulation as they strengthen the linear relaxation. Closest assignment constraints (23) are written separately per demand level and state that each demand level must be assigned to the closest facility of equal or higher level. Constraints (24) and (25) limit the number of new facilities to open and existing facilities to close. Constraints (26) limit the maximum travel distance between centers and facilities. Finally, constraints (27) define decision variables and enforce single assignment.

Closest assignment constraints (23) may be replaced by path assignment constraints, stated separately per demand level:

$\sum_{k \in P_{i j}} x_{k j s} \geqslant\left|P_{i j}\right| \cdot x_{i j s}, \quad \forall i \in I, j \in J, s \in S$.

Note that in constraints (28) the assignments of different levels are independent, while in constraints (23) the location of higher-level facilities influences lower-level assignments.

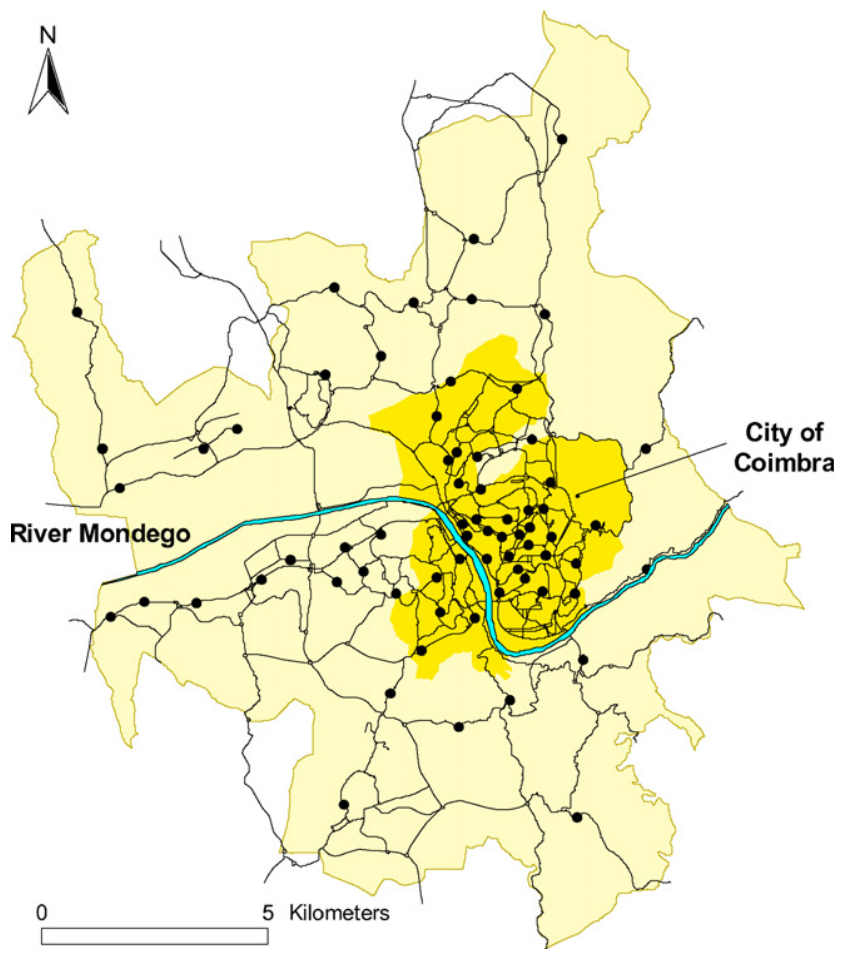

Fig. 2. Municipality of Coimbra, demand centers and road network. 
Table 2

Coimbra's school network

\begin{tabular}{llcc}
\hline School type & $\begin{array}{l}\text { Number of } \\
\text { schools }\end{array}$ & $\begin{array}{l}\text { Number of } \\
\text { classrooms }\end{array}$ & $\begin{array}{l}\text { Fraction of } \\
\text { capacity }(\%)\end{array}$ \\
\hline $\begin{array}{l}\text { Public EB1 } \\
\quad<4 \text { classrooms })\end{array}$ & 43 & 90 & 12 \\
$\begin{array}{l}\text { Public EB1 } \\
\quad(4+\text { classrooms })\end{array}$ & 28 & 149 & 20 \\
Private EB1 & 7 & 49 & 6 \\
Public EB12 & 9 & 258 & 34 \\
Private EB12 & 9 & 210 & 28 \\
Total & 96 & 756 & 100 \\
\hline
\end{tabular}

The formulation of model (HCM) given above allows facility co-location, i.e. the location of different types of facilities at the same site. This can advantageous to satisfy maximum capacity constraints. If co-location is not allowed then the following constraint should be added to the model:

$\sum_{t \in S} y_{j t} \leqslant 1, \quad \forall j \in J$

In addition, if co-location is not allowed, capacity constraints (20)-(22) can be replaced with the following simpler constraints:

$\sum_{t \in S} z_{j t}=\sum_{s \in S} \sum_{i \in I} u_{i s} x_{i j s}, \quad \forall j \in J$,

$z_{j t} \geqslant b_{j t} y_{j t}, \quad \forall j \in J, t \in S$,

$z_{j t} \leqslant B_{j t} y_{j t}, \quad \forall j \in J, t \in S$,

where constraints (30) define variables $z_{j t}$ as the total demand of all levels served at site $j$ by a type- $t$ facility, and constraints (31) and (32) impose minimum and maximum capacities. If (30)-(32) are used, constraints (19) are not redundant for the integer formulation and are needed to impose the nested facility hierarchy.

\section{Case study}

In this section, we present a study on the redeployment of Coimbra's primary school network. Coimbra is a municipality located in the center-littoral region of Portugal with a population of 150,000 inhabitants (Fig. 2). The primary school network of the municipality is composed of 96 schools (Table 2). Most of these schools are public, but there is a significant number of private schools. Some of them are fully subsidized by the government because they are located in areas not covered by public schools. There are two types of primary schools: EB1, for the first cycle of education; and EB12, for the first and the second cycles. The first cycle of education comprises 4 years and is attended by children aged $6-9$. The second cycle comprises 2 years and is attended by children aged 10 and 11. The EB1 school network consists of 78 schools with a total capacity of 288 classrooms (or 7200 students, assuming a maximum of 25 students per classroom). A large number of these schools has only one or two classrooms, which means that students of different years must share the same classrooms. The EB12 network consists of 18 schools with a total capacity of 468 classrooms (or 11,700 students). Following a period of fast decline in school age population, in 2004 the total number of enrollments in the primary schools of the municipality was 10,659 students. Since the aggregate capacity of these schools is 18,900 students, the aggregate occupation rate of the existing network was $56 \%$.

The study was made using the hierarchical capacitated median (HCM) model introduced in the previous section. Applications of location models to school network planning are numerous and we cite only some representative, relatively recent examples. Church and Murray (1993) present a multi-objective model considering school openings and closures and capacity balancing between schools. Pizzolato and Silva (1997) and Pizzolato et al. (2004) use a $p$-median model for clustering population centers. The clusters thus found are then analyzed by confronting total existing school capacity and population. Antunes and Peeters (2001) develop a dynamic location model where schools can be opened or closed, and their capacity can be expanded or reduced over time, with the objective of minimizing total discounted costs. Related models, where location decisions are not involved, address the short-term school network management problem of assigning students to existing schools. Church and Schoepfle (1993) describe a multi-objective model considering student preferences for schools and balancing of school capacity occupation and racial mix across schools. Caro et al. (2004) present a model for school districting contemplating several desired properties of school districts, including capacity balancing and contiguity. As far as we know, no school network planning study (or other public facility planning study) reported in the literature relied on a hierarchical model with capacity and assignment constraints.

Three objectives were pursued by the education authorities. First, school capacity should be adjusted 
to education demand (as noted above, current aggregate occupation rate is just $56 \%$ ). Second, accessibility of students to schools should be maximized. Third, changes to the existing network should be small, either because of scarce public budgets to build new schools or to avoid public reactions against school closure (particularly from parents and teachers). These objectives may of course be conflicting.

With regard to the first objective, following a decision by the education authorities, existing schools included in the study were current public schools with four classrooms or more and subsidized private schools. Other private schools, located in areas covered by public schools, which compete with public schools and are not subsidized, were left out of the study. Small public EB1 schools with less than four classrooms were also left out of the study, assuming these will be phased out in favor of larger, better equipped schools. In aggregate terms, the study included 25 EB1 schools with 4-10 classrooms and 14 EB12 schools with 15-36 classrooms, giving a total capacity of 149 and 367 classrooms, respectively. As projected demand in 2015 for the two cycles of education is 6300 and 3150 students, the aggregate occupation rate of the resulting school network would be $73 \%$.

The municipality of Coimbra was discretized in 68 population centers (Fig. 2). Sites were assumed to be coincident with centers. For this level of aggregation, eight centers contain both EB12 and EB1 schools. Travel was assumed to be made along the main road network, which is fully served by public transportation.
Three scenarios for the redeployment of the school network were considered. In Scenario 1, the minimum occupation of EB1 and EB12 schools was set to 40 and 120 students, respectively, to meet the guidelines of the Ministry of Education for school capacity (MinEdu, 2000). In Scenario 2, the minimum occupation of schools was increased to 80 students for EB1 schools and to $75 \%$ of current maximum capacity for EB12 schools. Scenario 3 is the same as Scenario 2, but allowing a new EB12 school to be opened, with a capacity between 360 and 600 students. The purpose of Scenarios 2 and 3 was to find the best way of adjusting the existing capacity to forecast demand, while keeping schools with good occupation. The maximum student-toschool travel distance was set to $8 \mathrm{~km}$ in all scenarios, also to meet the guidelines of the Ministry of Education.

We first solved model (HCM) with closest assignment constraints (23). For this model, no feasible solutions could be found for any one of the three scenarios, as these constraints are too "rigid" given the spatial distribution of existing capacity versus forecast demand. Then model (HCM) was used with path assignment constraints (28) replacing (23). The computation of data for these constraints was carried out in two main steps (recall that the pathassignment set $P_{i j}$ for a given center $i$ and site $j$ contains all centers "near" the travel path between $i$ and $j$ ). First, buffers around each center were created with a radius of half the distance to the nearest neighbor, truncated to a maximum of $1 \mathrm{~km}$, measured along the road network. Second, all centers whose buffer is intersected by the shortest path (on

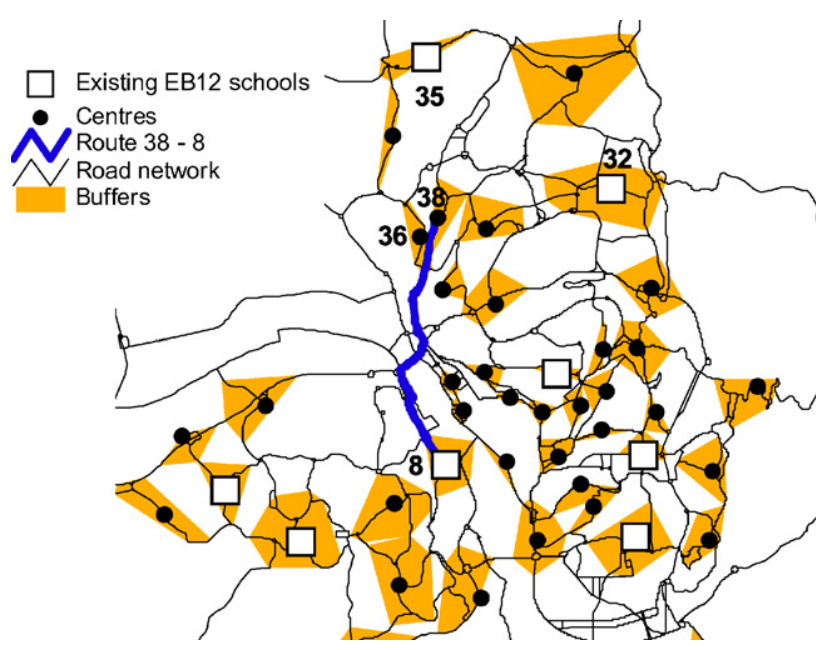

Fig. 3. Detail of path assignment. 
Table 3

Summary of solutions for the three scenarios

\begin{tabular}{|c|c|c|c|c|c|c|c|c|c|c|c|c|c|}
\hline \multirow[t]{3}{*}{ Scenario } & \multirow{2}{*}{\multicolumn{2}{|c|}{$\begin{array}{l}\text { Model } \\
\text { parameters }\end{array}$}} & \multirow{2}{*}{\multicolumn{2}{|c|}{$\begin{array}{l}\text { Total travel } \\
\text { distance }\end{array}$}} & \multirow{2}{*}{\multicolumn{3}{|c|}{$\begin{array}{l}\text { Aggregate school } \\
\text { occupation }\end{array}$}} & \multicolumn{6}{|c|}{ Number of schools } \\
\hline & & & & & & & & \multicolumn{2}{|c|}{ Total } & \multicolumn{2}{|l|}{ New } & \multicolumn{2}{|c|}{ Closed } \\
\hline & $q 1$ & $p 2$ & $\mathrm{~km}$ & Relative (\%) & EB1 $(\%)$ & EB12 (\%) & Total $(\%)$ & EB1 & EB12 & EB1 & EB12 & EB1 & EB12 \\
\hline 1 & 3 & 0 & 12,527 & 100 & 63 & 79 & 74 & 22 & 14 & 0 & 0 & 3 & 0 \\
\hline 2 & - & 0 & 14,391 & 115 & 78 & 90 & 88 & 13 & 13 & 0 & 0 & 12 & 1 \\
\hline 3 & - & 1 & 13,183 & 105 & 75 & 87 & 85 & 12 & 14 & 0 & 1 & 13 & 1 \\
\hline
\end{tabular}

the road network) from $i$ to $j$ were added to set $P_{i j}$. In the example of Fig. 3, $P_{38,8}=\{38,36,8\}$, which means that if center 38 is assigned to school 8 , then center 36 must be assigned to the same school.

The models were implemented with Dash Optimization's XPRESS-MP package. The modeling environment was XPRESS Mosel 1.4 (Dash, 2004) and the solver was XPRESS Optimizer version 15.30 (Dash, 2005), running under Windows XP on a computer with a Pentium-M 1.3 GHz CPU and $512 \mathrm{MB}$ of memory. All model runs took less than 3 minutes, as in this study the number of schools to open or close is relatively small. Arcview GIS 3 (ESRI,
2000) was used in conjunction with the optimizer for data handling and result analysis. Three programs were developed in Arcview's scripting language for the following purposes: (i) computing the distance matrix between all centers using the road network; (ii) computing sets $P_{i j}$ to be used with path assignment constraints; (iii) importing and displaying the solution output by the optimizer. The first two make use of Arcview's Network Analyst extension. In particular, the practical usefulness of path assignment constraints is closely tied to the ability to compute data with a real road network, and thus the use of a GIS is fundamental for this purpose.

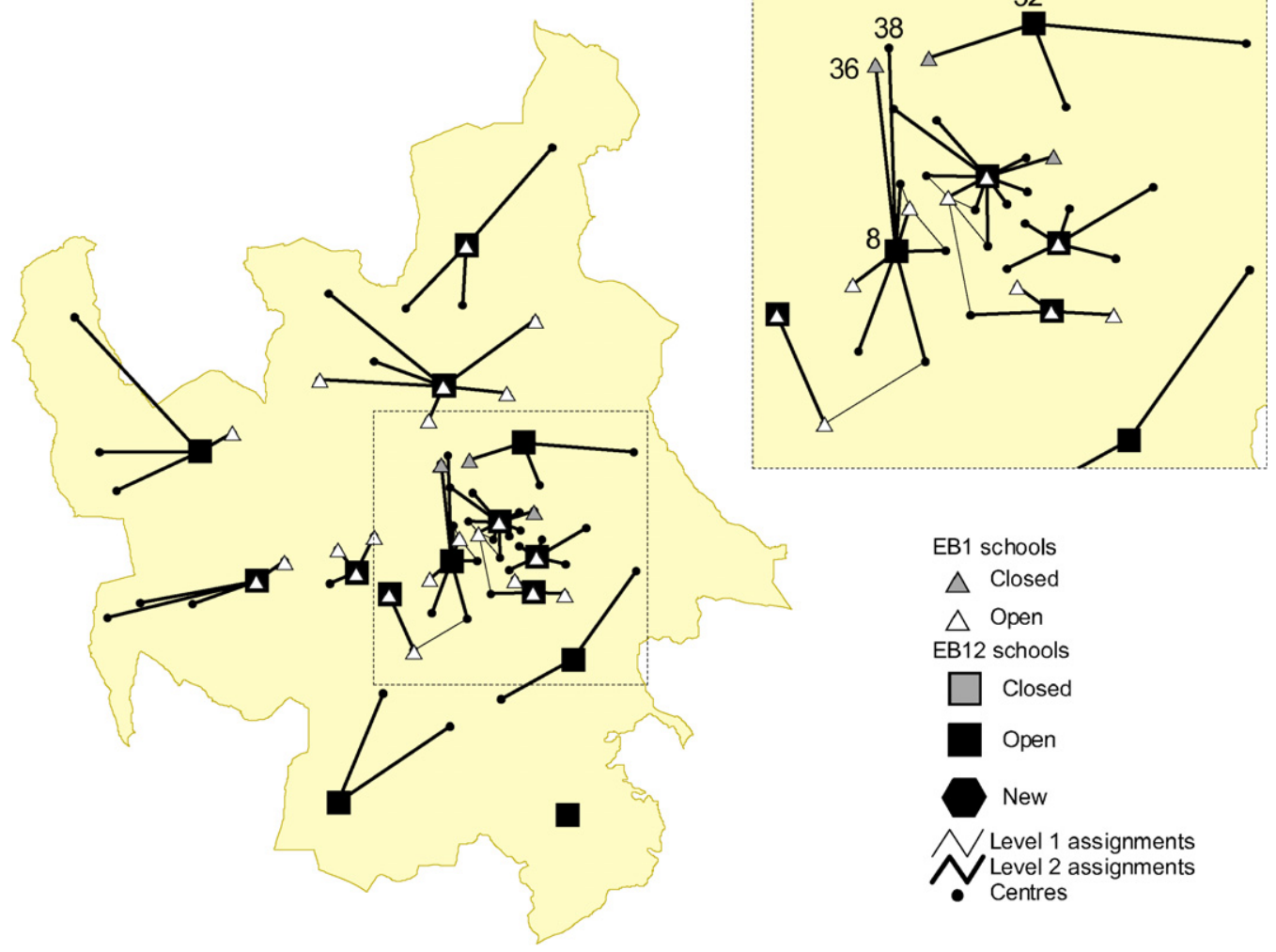

Fig. 4. Solution for Scenario 1. 

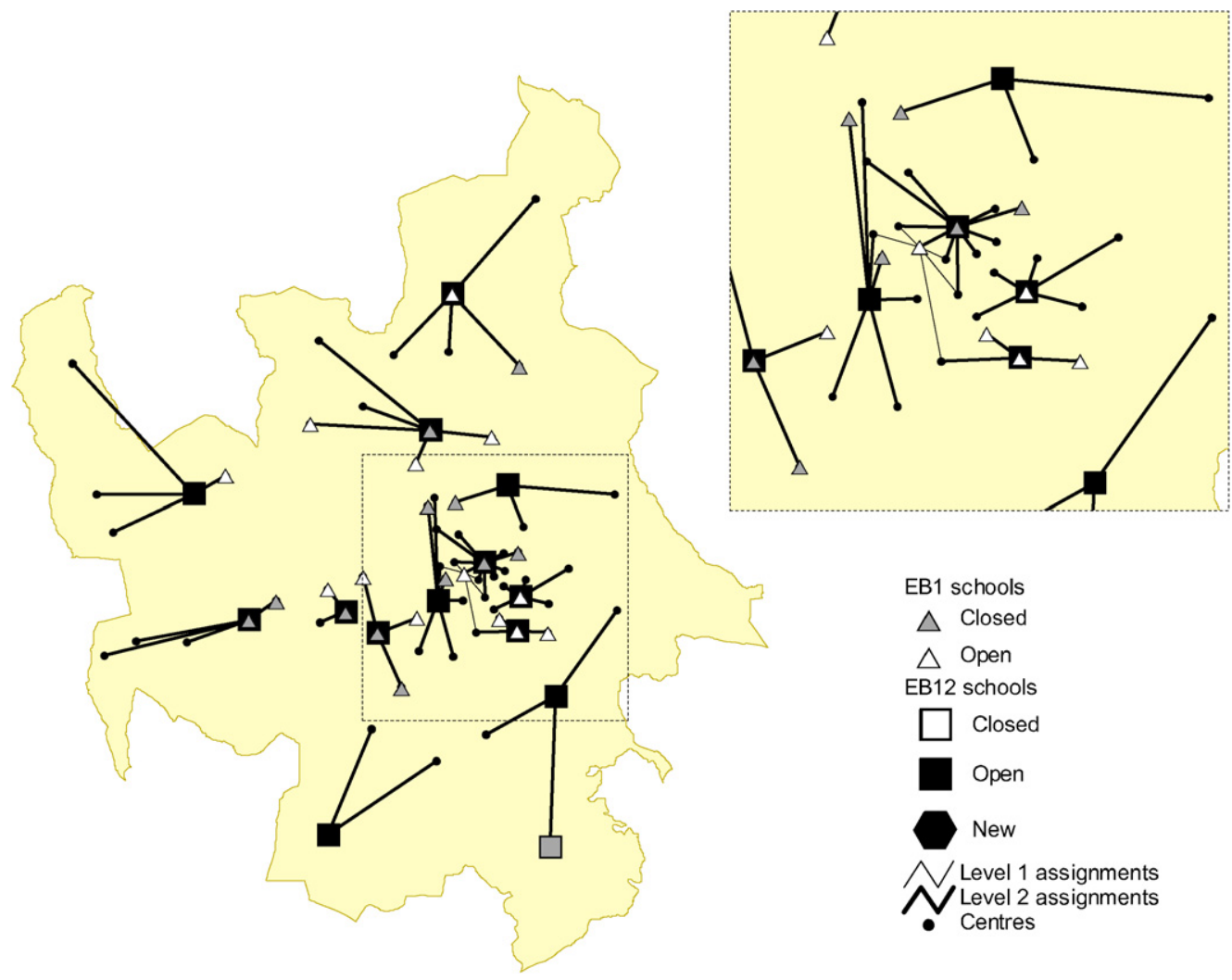

Fig. 5. Solution for Scenario 2.

Church (2002) discusses the link between GIS and the development and application of location models.

The solutions obtained for the three scenarios are summarized in Table 3, where parameters $q_{1}$ and $p_{2}$ are the maximum number of EB1 schools to close and EB12 schools to open, respectively (for all scenarios, no new EB1 schools could be open and any number of EB12 schools could be closed). The existing school network is sufficient for the future needs of the municipality (Scenario 1), with around 30\% of slack capacity. Three EB1 schools do not meet the minimum occupation requirements and are closed. A seemingly awkward assignment occurs for centers 36 and 38 (shown in Fig. 4, expanded in detail in Fig. 3), which are sent south to center 8 . However, the existing schools at centers 35 and 32 do not have enough capacity, and center 8 is less than $1 \mathrm{~km}$ further away than those schools. If the minimum occupation is augmented (Scenario 2), average occupation increases but total travel distance is degraded by $15 \%$, mainly because of the closure of one EB12 school in the south-east of the municipality (Fig. 5). In this scenario, 12 EB1 schools are closed. If a new school is allowed (Scenario 3), high average occupations are still guaranteed and travel distance is only $5 \%$ higher than in Scenario 1 (Fig. 6). The main beneficiaries are centers 36 and 38, located in a fast growth area currently not covered satisfactorily.

\section{Conclusion}

In this article, we presented a discrete hierarchical location model for public facility planning. The main features of the model are: an accessibilitymaximization objective; several levels of demand; several types (or levels) of facilities; a nested hierarchy of facilities (i.e. a facility of a given level can serve demand of equal and lower levels); maximum and minimum capacity (or occupation) constraints; and user-to-facility assignment constraints. The latter include single-assignment and closest-assignment constraints, as well as a new type of constraints called path-assignment constraints. They are used to enforce some desirable properties for the spatial pattern of assignments. The resulting solutions are 


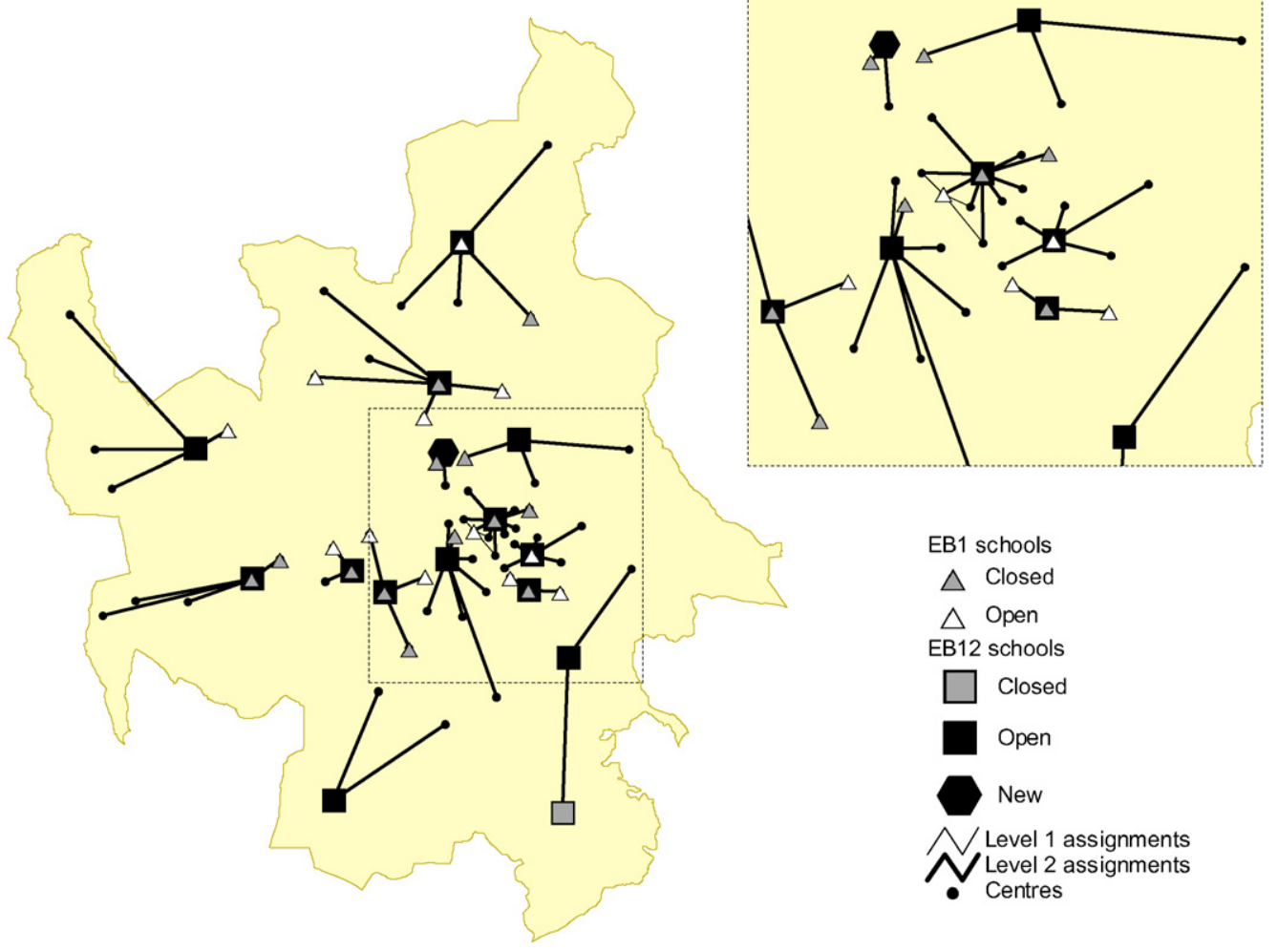

Fig. 6. Solution for Scenario 3.

easier to interpret and to explain in a public facility planning context, therefore being more likely to be accepted by the users. As far as the authors know, a model with this set of features has never been dealt with in the literature.

The hierarchical location model was developed to help in a real-world school network planning process conducted for the municipality of Coimbra, Portugal. At present, most public planning processes include complex participatory mechanisms. These mechanisms started to be introduced in the late $80 \mathrm{~s}$, as a reaction to the alleged failure of rational, model-based approaches to public planning (Chadwick, 1978). According to many planning theorists, a new type of approach was needed focusing on participation and debate rather than on rationality and modeling (Healey, 1992). This new type of approach was progressively adopted and now underlies many real-world planning processes. What we clearly realized from our involvement in the preparation of the Coimbra Educational Charter is that debate and modeling, instead of being substitutes, are close complements. Indeed, in the presence of stakeholders with differ- ent viewpoints and interests, like those represented in the education council of the municipality, objectives can be debated and agreed upon, but solutions that are not rational for the objectives retained are too fragile to prevail. When the number of possible solutions is very large, the only way of finding the rational solutions for a planning problem involves the application of optimization models. Without the hierarchical location model we developed, it would have been extremely difficult to arrive at planning solutions widely accepted by the Education Council.

The hierarchical location model was solved with a modern commercial optimizer rather easily. The use of a GIS package in conjunction with the optimization program was extremely valuable. In addition to simplify the analysis of results, the GIS was used for computing data for path assignment constraints, using features such as finding shortest paths and create buffers, or areas of influence, around centers with distances measured on the road network. The model was easy to solve because Coimbra, though being one of the largest municipalities in Portugal outside the metropolitan areas 
of Lisbon and Porto, still is a small-size municipality by European standards, and also because small changes in the number of schools were allowed. If any one of these conditions were not met, the optimization program would have been unable to do the job. Our future research will be directed towards the development of specialized solution procedures for solving large-scale hierarchical and (non-hierarchical) capacitated models with user-to-facility assignment constraints still out of reach of current modern commercial optimizers.

\section{References}

Antunes, A., Peeters, D., 2001. On solving complex multi-period location models using simulated annealing. European Journal of Operational Research 130 (1), 190-201.

Caro, F., Shirabe, T., Guignard, M., Weintraub, A., 2004. School redistricting: Embedding GIS tools with integer programming. Journal of the Operational Research Society 55, 836849.

Carreras, M., Serra, D., 1999. On optimal location with threshold requirements. Socio-economic Planning Sciences 33 (2), 91103.

Chadwick, G., 1978. A Systems View of Planning. Pergamon Press, Oxford, UK.

Church, R.L., 2002. Geographical information systems and location science. Computers and Operations Research 29 (6), 541-562.

Church, R.L., Eaton, D.J., 1987. Hierarchical location analysis using covering objectives. In: Ghosh, A., Rushton, G. (Eds.), Spatial Analysis and Location-Allocation Models. Van Nostrand Reinhold, New York.

Church, R.L., Murray, T., 1993. Modeling school utilization and consolidation. Journal of Urban Planning and Development 119 (1), 23-38.

Church, R.L., Schoepfle, O.B., 1993. The choice alternative to school assignment. Environment and Planning B 20 (4), 447 457.

Dash Optimization, Ltd., 2004. XPRESS-Mosel Language Reference Manual - Release 1.4.

Dash Optimization, Ltd., 2005. XPRESS-Optimizer Reference Manual - Release 15.

Daskin, M.S., 1995. Network and Discrete Location: Models, Algorithms, and Applications. Wiley Interscience, New York.

Eitan, Y., Narula, S.C., Tien, J.M., 1991. A generalized approach to modeling the hierarchical location-allocation problem. IEEE Transactions on Systems, Man, and Cybernetics 21 (1), 39-46.

ESRI - Environmental Systems Research Institute, 2000. Using Arc View GIS, Redlands, CA.

Galvão, R.D., Espejo, L.G.A., Boffey, B., Yates, D., 2006. Load balancing and capacity constraints in a hierarchical location model. European Journal of Operational Research 172, 631646.

Gerrard, R.A., Church, R.L., 1996. Closest assignment constraints and location models: Properties and structure. Location Science 4 (4), 251-271.

Healey, P., 1992. Planning through debate: The communicative turn in planning theory. Town Planning Review 63 (2), 143162.

Kalcsics, J., Melo, M.T., Nickel, S., Gündra, H., 2002. Planning sales territories - A facility location approach. In: Operations Research Proceedings 2001. Springer Verlag, Berlin, pp. 141148.

Krarup, J., Pruzan, P.M., 1983. The simple facility location problem: Survey and synthesis. European Journal of Operational Research 12, 36-81.

Labbé, M., Louveaux, F.V., 1997. Locations problems. In: Dell'Amico, M., Maffioli, F., Martello, S. (Eds.), Annotated Bibliographies in Combinatorial Optimization. Wiley, Chichester, UK, pp. 61-281.

Marianov, V., Serra, D., 2002. Location problems in the public sector. In: Drezner, Z., Hamacher, H. (Eds.), Facility Location: Applications and Theory. Springer-Verlag, Berlin, Germany, pp. 119-150.

Mehrotra, A., Johnson, E.L., Nemhauser, G.L., 1998. An optimization based heuristic for political districting. Management Science 44 (8), 1100-1114.

MinEdu - Ministério da Educação, 2000. Critérios de Reordenamento da Rede Educativa (Criteria for the Redeployment of School Networks), Portugal.

Narula, S., 1986. Minisum hierarchical location-allocation problems on a network: A survey. Annals of Operations Research 6, 257-272.

Pizzolato, N., Silva, H., 1997. The location of public schools: Evaluation of practical experiences. International Transactions in Operational Research 4 (1), 13-22.

Pizzolato, N.D., Barcelos, F.B., Lorena, L.A.N., 2004. School location methodology in urban areas of developing countries. International Transactions in Operational Research 11, 667681.

ReVelle, C., 1987. Urban public facility location. In: Mills, E. (Ed.), Handbook of Regional and Urban Economics, vol. II. Elsevier, Amsterdam, The Netherlands, pp. 1053-1096.

ReVelle, C.S., Eiselt, H.A., 2005. Location analysis: A synthesis and survey. European Journal of Operational Research 165 (1), 1-19.

ReVelle, C.S., Swain, R.W., 1970. Central facilities location. Geographical Analysis 2 (1), 30-42.

Verter, V., Lapierre, S., 2002. Location of preventive health care facilities. Annals of Operations Research 110, 123-132.

Weaver, J.R., Church, R.L., 1991. The nested hierarchical median facility location model. INFOR 29, 100-115.

Weisstein, E.W., 1999. Delaunay triangulation, MathWorld - A Wolfram Web Resource, http://mathworld.wolfram.com/ DelaunayTriangulation.html (retrieved 18 April 2006).

Zoltners, A.A., Sinha, P., 1983. Sales territory alignment: A review and model. Management Science 29, 1237-1256. 